\title{
Luminosity Function of Galaxies in Substructures of Galaxy Clusters
}

\author{
Janusz Krywult \\ Institute of Physics, Swietokrzyska Academy, Kielce, Poland \\ email: krywult@pu.kielce.pl
}

\begin{abstract}
The analysis of the R-band luminosity function of galaxies in substructures of 21 Abell clusters shows that the characteristic magnitude $M^{*}$ for dominant substructure is brighter than in the second substructure and the magnitude gap between two brightest galaxies in dominant substructure is larger than in the second one.
\end{abstract}

Keywords. Galaxies, clusters, luminosity function, substructures

The luminosity function (LF) of galaxies plays an important role in the analysis of the properties of galaxies. Because the shape of the galaxy luminosity function varies with environment, the clusters of galaxies are good systems to test theories of galaxy formation and evolution.

The results of the 2-D wavelet analysis (Flin \& Krywult 2006) was served as a basis of this study. We selected clusters having only two statistically significant substructures detected by the wavelet analysis. The first $(a)$ and the second $(b)$ substructure in each cluster has comparable number, but more than 10, of galaxies.

The Schechter (1976) integrated LF was fitted to unbinned luminosities of galaxies in each analyzed substructure by $\chi^{2}$ method with $M^{*}$ and $\phi^{*}$ as a free parameters. The faint-end slope was fixed to the universal value $\alpha=-1.25$. The first ranked galaxy in the substructure was excluded from the fitting procedure.

To eliminate uncertainties associated with calibration the magnitude $M_{10}$ of the 10-th ranked galaxy in substructure was used as a reference point. The mean value of the difference between magnitudes of the 10-th ranked galaxies in both substructures of the cluster $M_{a 10}-M_{b 10}=-0.17 \pm 0.11$.

The analysis of the luminosity function and brightest galaxies in the substructures of the sample of 21 Abell clusters shows as follows:

- The correlation between magnitude of the first-ranked galaxy and the characteristic luminosity for galaxies in both substructures is positive $\left(r_{a}=0.68, r_{b}=0.81\right)$.

- The $\chi^{2}$ test shows correlation between $\left(M_{a 1}-M_{a 10}\right)$ and $\left(M_{b 1}-M_{b 10}\right)$ at the significance level $\alpha=0.05$.

- The mean value of the gap between two brightest galaxies in dominated substructures is equal $M_{a 1}-M_{a 2}=-0.52 \pm 0.11$. For the second substructures $M_{b 1}-M_{b 2}=-0.38 \pm 0.10$.

- The characteristic magnitude $M_{a}^{*}$ is brighter than $M_{b}^{*}$ by $0.28 \pm 0.18$ magnitude.

The study seems to indicate differences on the evolution of galaxies in dominated and second substructure of galaxy clusters.

\section{References}

Flin, P., \& Krywult, J. 2006, AA 405, 9.

Schechter, P. 1976, ApJ 203, 297. 